\title{
The Earthquake Arrest Zone
}

\author{
Chun-Yu Ke ${ }^{1}$, Gregory C. McLaskey ${ }^{1}$, David S. Kammer ${ }^{2}$ \\ ${ }^{1}$ School of Civil and Environmental Engineering, Cornell University, Ithaca, New York, USA \\ ${ }^{2}$ Institute for Building Materials, ETH, Zürich, Switzerland
}

\begin{abstract}
This is a pre-copyedited, author-produced PDF of an article accepted for publication in Geophysical Journal International following peer review. The version of record is available online at: http://doi.org/10.1093/gji/ggaa386.
\end{abstract}

\section{Keywords:}

- Friction

- Mechanics, theory, and modelling

Corresponding author: David S. Kammer, dkammer@ethz.ch 


\begin{abstract}
Earthquake ruptures are generally considered to be cracks that propagate as fracture or frictional slip on preexisting faults. Crack models have been used to describe the spatial distribution of fault offset and the associated static stress changes along a fault, and have implications for friction evolution and the underlying physics of rupture processes. However, field measurements that could help refine idealized crack models are rare. Here we describe large-scale laboratory earthquake experiments, where all rupture processes were contained within a 3-m long saw-cut granite fault, and we propose an analytical crack model that fits our measurements. Similar to natural earthquakes, laboratory measurements show coseismic slip that gradually tapers near the rupture tips. Measured stress changes show roughly constant stress drop in the center of the ruptured region, a maximum stress increase near the rupture tips, and a smooth transition in between, in a region we describe as the earthquake arrest zone. The proposed model generalizes the widely used elliptical crack model by adding gradually tapered slip at the ends of the rupture. Different from the cohesive zone described by fracture mechanics, we propose that the transition in stress changes and the corresponding linear taper observed in the earthquake arrest zone are the result of rupture termination conditions primarily controlled by the initial stress distribution. It is the heterogeneous initial stress distribution that controls the arrest of laboratory earthquakes, and the features of static stress changes. We also performed dynamic rupture simulations that confirm how arrest conditions can affect slip taper and static stress changes. If applicable to larger natural earthquakes, this distinction between an earthquake arrest zone (that depends on stress conditions) and a cohesive zone (that depends primarily on strength evolution) has important implications for how seismic observations of earthquake fracture energy should be interpreted.
\end{abstract}

\title{
1 Introduction
}

The slip profile of an earthquake rupture is the spatial distribution of displacement discontinuity between the fault surfaces, $\delta(x)$. The slip profile of a single earthquake is directly related to the spatial distribution of on-fault stress changes associated with the rupture, $\Delta \tau(x)$. It is therefore important for understanding the mechanics of earthquakes and has implications for stress drop, stress redistribution, and earthquake-to-earthquake triggering (Freed, 2005). 
Most analytical models of slip profiles are mathematically convenient but can produce physically unrealistic results. Earthquakes are commonly modeled as shear cracks, and the linear elastic crack model (Bilby \& Eshelby, 1968) established that a perfect crack with uniform shear stress drop within the rupture area leads to an elliptical slip profile (Fig. 1a). This "elliptical model" casts an infinite stress increase at the rupture tips, which is unrealistic because real interfaces have finite strength. Cowie and Scholz (1992) and Bürgmann et al. (1994) assumed perfectly plastic failure near the rupture tips by adapting the Dudgale (1960) model to a mode II crack. The resulting "bell-shaped" model, shown in Fig. 1a, assumes a constant stress drop inside the ruptured region and a constant stress increase near the rupture tips (Fig. 1c). However, in our experiments, we did not observe a constant stress increase near the rupture tips.

Most past field studies of fault slip distributions provide information relevant to the growth of brittle faults over many earthquakes or slow slip events. Studies of faulting showed that slip gradients appeared approximately constant near the fault tip (Muraoka \& Kamata, 1983; Walsh \& Watterson, 1987; Dawers et al., 1993; Nicol et al., 1996; Manighetti et al., 2001), typically $20 \%$ of the rupture length (Cowie \& Scholz, 1992; Scholz \& Lawler, 2004). When considering slip profiles from individual events, measured slip distributions are often so heterogeneous that stacking of many individual events is required to evaluate features. Using this approach, Manighetti et al. (2005) found that slip distributions derived from kinematic models and field observations were roughly triangular and predominantly asymmetric. Walsh and Watterson (1987) argued that the ubiquitous linear tapering feature of slip profiles can be the result of cumulative slips from multiple growing cracks with elliptical shape. This argument highlights the difficulty of distinguishing the field measurements of slip profiles accumulated across multiple earthquake ruptures and of a single earthquake rupture, which will result in very different shapes and possibly different conclusions.

Slip at the rupture tips is small and difficult to measure, but can have a strong influence on stress changes. In this work, we use measurements of laboratory earthquakes to illuminate the features of earthquake slip profiles, including the area near the rupture tip. We present results from recent large-scale laboratory experiments where the rupture processes are partially or completely contained in a 3-meter long saw-cut granite fault (Ke et al., 2018; Wu \& McLaskey, 2019; McLaskey, 2019). This provides a unique opportunity to measure local slip and local static shear stress changes near the tip of an arrested rupture. Similar 
to observations from natural fault ruptures, we consistently observe slip profiles that taper approximately linearly.

In this work, we define the "earthquake arrest zone" as a subsection of an earthquake's rupture area. It is bounded on one side by the tip of an arrested earthquake rupture. The boundary on the other side is not as clearly defined, but is roughly located where the stress changes that occur during the earthquake $\Delta \tau(x)>\Delta \tau_{\min }$, as shown in Fig. 1d. When a propagating rupture enters the earthquake arrest zone, the rupture front decelerates and ultimately arrests. The elliptical model has an earthquake arrest zone width $w_{\mathrm{az}}=0$, and the bell-shaped model has a finite $w_{\mathrm{az}}$ with constant stress changes within the arrest zone. In our experiments, we find $w_{\mathrm{az}}$ on each end of the rupture is approximately $20 \%$ of the overall rupture length, and within each earthquake arrest zone we observe stress changes that gradually transition from a peak at the rupture tip to a minimum within the interior of the ruptured region. We propose an analytical crack model that accommodates the aforementioned observations and adheres to physical constraints better than previous models.

For each crack model, stress changes can be plotted against slip, as shown in Fig. 1e. Our proposed model produces a relationship that, on first glance, appears to be similar to a linear slip weakening relationship (e.g., Ida, 1972; Palmer \& Rice, 1973; Andrews, 1976). However, Fig. 1e shows the final slip and static stress changes at many different locations throughout the earthquake arrest zone, and this is different from a linear slip weakening relationship which describes the evolution of frictional strength as a function of slip at one location on the fault. As will be shown in this work, the earthquake arrest zone is fundamentally different from the cohesive zone defined in fracture mechanics (e.g., Freund, 1990; Day, Dalguer, Lapusta, \& Liu, 2005) due to its physical interpretation. The cohesive zone depends primarily on fault strength evolution (friction), but we will demonstrate that the earthquake arrest zone is produced by the heterogeneous initial stress distribution required to stop the earthquake rupture (see Section 6.3). Section 6.4 presents dynamic rupture simulations that confirm how arrest conditions affect the slip profile and static stress changes during an earthquake (and consequently, the $\Delta \tau-\delta$ relationships shown in Fig. 1e) but are largely independent of frictional strength.

The difference between a cohesive zone and an earthquake arrest zone has implications for how seismically observed earthquake fracture energy $E_{\mathrm{G}}$ should be interpreted. Here 
we draw a distinction between $E_{\mathrm{G}}$-referred to simply as "fracture energy" in seismology, or "breakdown energy/work" in previous studies (Abercrombie \& Rice, 2005; Viesca \& Garagash, 2015; Cocco et al., 2016; Perry et al., 2020) — and $\Gamma$ the fracture energy normally used in fracture mechanics (e.g., Andrews, 1976; Day et al., 2005). $\Gamma$ is a local property of the material or interface that depends on local strength evolution $\tau_{\mathrm{s}}(\delta)$ according to $\Gamma=\int_{0}^{\infty}\left[\tau_{\mathrm{s}}(\delta)-\tau_{\mathrm{r}}\right] d \delta$ (Rice, 1968; Ida, 1972), in which $\tau_{\mathrm{r}}$ is the residual strength. ( $\Gamma$ is a constant in our numerical simulations presented in Section 6.4 that employ linear slip weakening friction.) $E_{\mathrm{G}}$ is the total strain energy released during the earthquake minus the radiated energy $E_{\mathrm{R}}$ and the frictional work on the fault plane $E_{\mathrm{F}}$ (Kanamori \& Rivera, 2006). It has been assumed that $E_{\mathrm{G}}$ derived from properties of seismic waves can be related to the strength of the interface or intact rocks (e.g., Abercrombie \& Rice, 2005. However, we suggest that the estimation of $E_{\mathrm{G}}$ can be greatly affected by rupture and arrest properties and is largely independent of fault strength and $\Gamma$.

\section{Experimental Methods and Measurements}

Experiments were conducted on a biaxial direct shear apparatus as shown in Fig. 2. Slip events occurred on the simulated fault as shear load increased. The dimensions of the moving block and the stationary block are $3.10 \mathrm{~m} \times 0.81 \mathrm{~m} \times 0.30 \mathrm{~m}$, and $3.15 \mathrm{~m} \times 0.61 \mathrm{~m}$ $\times 0.30 \mathrm{~m}$ (respectively) in the $x, y$, and $z$ directions. The dimensions of the simulated fault are $3.10 \mathrm{~m} \times 0.30 \mathrm{~m}$ with area $A=0.95 \mathrm{~m}^{2}$. The fault surfaces of the granite samples were prepared by the manufacturer to be flat and parallel to $125 \mu \mathrm{m}$. Mechanical properties of the Barre Gray granite are $E=30 \mathrm{GPa}$ and $\nu=0.23$.

The normal loading array, consisting of $18 \times 2$ hydraulic cylinders, presses the two rock blocks together in the $y$-direction and applies normal contact pressure on the fault. The shear loading array, consisting of $6 \times 3$ hydraulic cylinders, pushes the moving block in $+x$-direction and applies shear stress on the fault. Hydraulic cylinders in each array are interconnected to a manual pump, allowing us to independently control normal and shear loading. The measurements of hydraulic pressure in both arrays are then converted and reported as sample average normal and shear stress, $\bar{\sigma}$ and $\bar{\tau}$.

Local fault slip was measured by 16 evenly spaced eddy current displacement sensors at 16 locations $\left(\mathrm{E}_{1}-\mathrm{E}_{16}\right)$ along the fault as shown in Fig. 2. These sensors measure the relative displacement between each side of the fault, i.e., the moving rock block and the stationary 

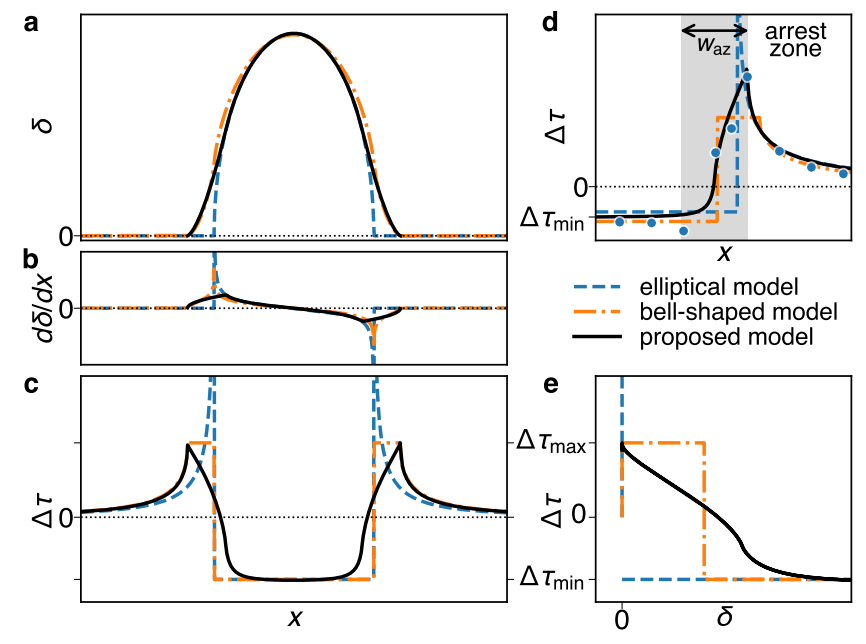

Figure 1. Examples of (a) slip profile $\delta(x)$, (b) first derivative of slip profile $d \delta(x) / d x$, (c) associated shear stress changes $\Delta \tau(x)$. The bell-shaped model is designed to limit the maximum shear stress, casting a constant $\Delta \tau$ plateau near rupture tips under uniform loading and strength field. The proposed model preserves the elliptical slip profile in the center and swaps the edges of the crack with $x^{3 / 2}$ form, eliminates shear stress singularities, keeps $\Delta \tau(x)$ peaks at rupture tips, and produces a smooth distribution of stress changes with the earthquake arrest zone. (d) a zoom-in of (c) shows the earthquake arrest zone (shaded) in more detail and presents example laboratory measurements (blue dots) of near-fault stress changes with fitted models. $w_{\text {az }}$ denotes earthquake arrest zone width. Two distinct data measurements lie inside the earthquake arrest zone where stress changes transition from an apparent maximum level, $\Delta \tau_{\max }$, at the crack tip to a minimum level, $\Delta \tau_{\min }$, inside the crack. (e) depicts the earthquake stress change versus slip, $\Delta \tau-\delta$, relationship of all three models. 


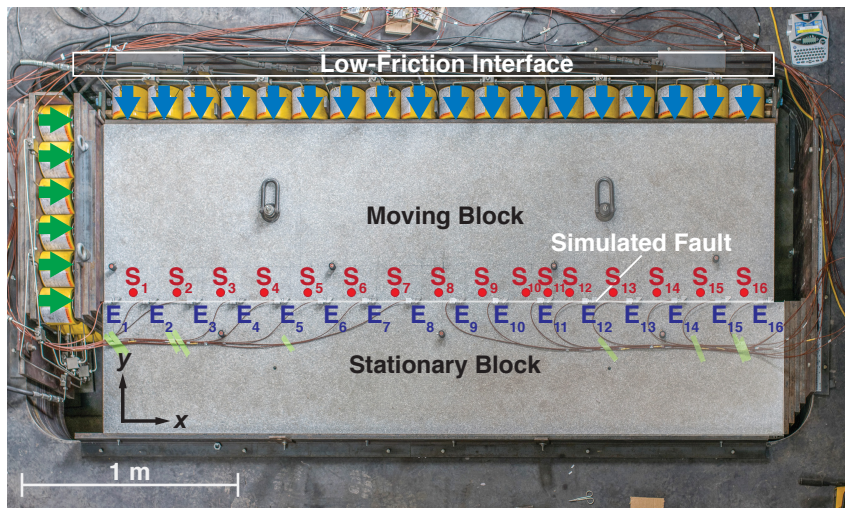

Figure 2. Experimental setup. The moving block and the stationary block were pressed together to compose the simulated fault of granite. A low friction interface consisted of a $2.4 \mathrm{~mm}$ thick sheet of reinforced Teflon sliding on precision ground steel plates $(\mu \approx 0.1)$ allows the normal loading array (blue arrows) to translate with the moving block in the $x$-direction. The shear loading array (green arrows) pushes the moving block in the $x$-direction to apply shear stress and induce ruptures on the simulated fault. $\mathrm{S}_{1}-\mathrm{S}_{16}$ show the locations of 16 sets of strain gage pairs and $\mathrm{E}_{1}-\mathrm{E}_{16}$ show the locations of 16 slip sensors.

rock block. Local shear strain was measured by 16 pairs $\left(\mathrm{S}_{1}-\mathrm{S}_{16}\right)$ of semiconductor strain gages at locations shown in Fig. 2, with $\mathrm{S}_{11}$ and $\mathrm{E}_{11}$ being collocated and all others evenly spaced between $\mathrm{E}_{1}-\mathrm{E}_{16}$. Each pair consists of two collocated $4 \mathrm{~mm}$ long semiconductor strain gages oriented at $45^{\circ}$ and $135^{\circ}$ from the fault which were glued to the moving block, $5 \mathrm{~mm}$ from the fault. Local shear stress $\tau$ was derived from measurements of the strain gage pair and the elastic properties of Barre Gray granite. While the $5 \mathrm{~mm}$ off-fault measurement can be biased for dynamic responses (Svetlizky \& Fineberg, 2014; Kammer \& McLaskey, 2019; Svetlizky et al., 2020), we assume negligible differences between on-fault and $5 \mathrm{~mm}$ off-fault measurements when at (quasi-)static stress states.

Before every experiment, we apply $\bar{\sigma} \approx 1 \mathrm{MPa}$ and then increase $\tau$ until the whole simulated fault slips a few times to create a consistent initial stress distribution for the following procedures. During the experiments, normal load was first increased to the prescribed level $\bar{\sigma}_{0}$ and a valve was closed to keep the volume of hydraulic fluid in the normal loading array constant. Shear load was then increased at a roughly constant rate to induce sequences of slip events. Further information about the experimental setup, procedures, and mechanics of the sequences can be found in Ke et al. (2018) and Wu and McLaskey (2019). 
In this work, we study individual coseismic slip events. In our experiments, slow fault creep and nucleation-related slow slip sometimes occurs prior to and after slip events, as shown in Fig. 3a. For these events, using a smaller time window to calculate $\delta$ and $\Delta \tau$ could exclude quasi-static nucleation process and result in incomplete $\delta(x)$ and $\Delta \tau(x)$, as shown in Fig. 3b. On the other hand, using a larger time window that includes the nucleation process and afterslip will also include stress changes from the slow and continuous loading and slip from quasi-static steady slow slip. We account for these slow processes by fitting linear trends in time histories before and after the dynamic rupture process then extrapolating the linear time histories to the instant of the dynamic rupture process and we then take differences to define the $\delta$ and $\Delta \tau$ associated with a dynamic slip event from each location (Fig. 3). In our experiments, rapid afterslip appears to slightly decrease the stress increase at the rupture tip of arrested ruptures (not shown), and likely accounts for only a $5 \%$ change. The above procedure lumps the slow slips prior to and after the dynamic rupture to the changes between the static states before and after the event. Events with fast nucleation and no afterslip are unaffected.

\section{Spatial Distribution of Stress Changes}

For a mode II (in-plane shear) crack, we define the spatial distribution of shear stress change associated with an earthquake rupture as $\Delta \tau(x) \equiv \tau_{\mathrm{f}}(x)-\tau_{0}(x)$, where $\tau_{0}(x)$ is the spatial distribution of shear stress at the (quasi-)static state before the rupture nucleates and $\tau_{\mathrm{f}}(x)$ is the spatial distribution of shear stress at the (quasi-)static state after the rupture arrests. Thus, $\Delta \tau(x)$ is the shear stress changes due to all processes of a rupture (nucleation, dynamic rupture propagation, and rapid afterslip) between two (quasi-)static states. Bilby and Eshelby (1968) derived the constitutive relationship between the distribution of slip parallel to the fault $\delta(x)$ and shear stress change distribution $\Delta \tau(x)$,

$$
\Delta \tau(x)=-\frac{\mu^{*}}{2 \pi} \int_{a_{-}}^{a_{+}} \frac{d \delta(\xi) / d \xi}{x-\xi} d \xi
$$

where $\mu^{*}=\mu /(1-\nu)$ for mode I and II, $\mu^{*}=\mu$ for mode III, in which $\mu$ is the shear modulus and $\nu$ is the Poisson's ratio, and $a_{ \pm}$are the locations of the rupture tips. This equation assumes the material surrounding the rupture is linear elastic. It takes the first derivative of the slip profile $d \delta(x) / d x$ as input and gives its respective static stress change distribution $\Delta \tau(x)$. Note that if a given $\delta(x)$ is $C^{1}$ continuous and $\delta(x) \sim\left( \pm\left[a_{ \pm}-x\right]\right)^{3 / 2}$ as $x$ approaches $a_{ \pm}$within the rupture, its respective $\Delta \tau(x)$ is smooth and finite (see Uenishi and Rice (2003): Appendix A). 

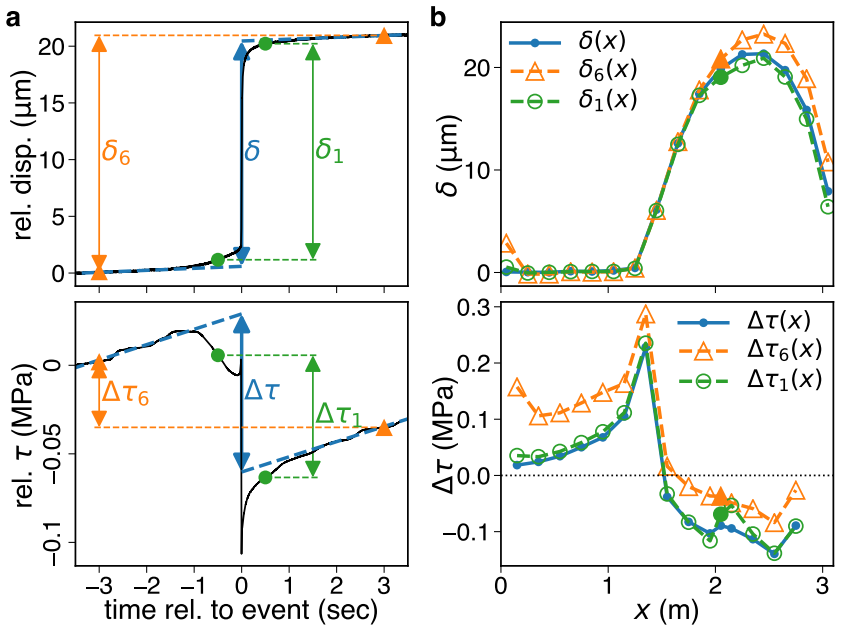

Figure 3. Example of slip and stress change time histories and extracted $\delta$ and $\Delta \tau$ from FS01-038-7MPa-P-1-03 event. (a) Heavy dashed lines are linear trends associated with continuous loading and fitted from data before $(t=-3$ to $-2 \mathrm{sec})$ and after $(t=2$ to $3 \mathrm{sec})$ the event. Parameters $\delta$ and $\Delta \tau$ are then defined by the difference between linear trends before and after the event extrapolated to the instant of dynamic rupture $(t=0)$ as shown. $\delta_{6}$ and $\Delta \tau_{6}$ are defined by the difference with a 6 -second time window, i.e., difference between $t= \pm 3$ second. Similarly, $\delta_{1}$ and $\Delta \tau_{1}$ are defined by the difference with a 1-second time window. (b) Solid curves are results of $\delta(x)$ and $\Delta \tau(x)$ with linear trends removed. Dashed curves are estimates made without linear trends removed. The estimate from a 6 -s window, $\delta_{6}(x)$, is slightly larger than $\delta_{1}(x)$ due to the inclusion of quasi-static slip during nucleation and after slip. Similarly, $\Delta \tau_{6}>\Delta \tau_{1}(x)$ due to the inclusion of stress changes associated with continuous loading. Note that the deviations near $x=$ $2 \mathrm{~m}$ in both $\delta_{1}(x)$ and $\Delta \tau_{1}(x)$ were due to the exclusion of the quasi-static nucleation process. 


\section{Proposed Crack Model}

Our model combines the elliptical shape in the center of the rupture and an $r^{3 / 2}$ form at the edges, which replaces stress singularities in the elliptical model with mathematically simplistic earthquake arrest zones. The edges of the slip profile are approximately linear (Fig. 1a), consistent with slip profiles obtained from natural faults. The proposed analytical model of slip profiles is formulated as

$$
\delta(r)= \begin{cases}D\left[1-\left(\frac{r}{\lambda a}\right)^{2}\right]^{1 / 2} & , 0 \leq r \leq r^{\text {joint }} \\ \delta^{\text {joint }}\left(\frac{r-a}{r^{\text {joint }}-a}\right)^{3 / 2} & , r^{\text {joint }}<r \leq a \\ 0 & , a<r\end{cases}
$$

where $r$ is the distance to the center of the crack, $a$ is the radius of the crack, $\lambda$ scales $a$ to the radius of the ellipse $a^{\text {ellipse }}=\lambda a$, in which $0<\lambda<1, r^{\text {joint }}=a\left(\sqrt{1+3 \lambda^{2}}-1\right)$ is the radius where $\delta(r)$ switches between elliptical and $r^{3 / 2}$ form, and $\delta^{\text {joint }}=\delta\left(r^{\text {joint }}\right)$, as shown in Fig. 4a. Compared to the elliptical (or ellipsoidal) model, $\delta(r)=D\left[1-\left(\frac{r}{a}\right)^{2}\right]^{1 / 2}$ for $0 \leq r \leq a$, this model introduces only one additional parameter, $\lambda$, and guarantees $C^{1}$ continuity in $\delta(r)$ and no singularity in the associated stress changes if $0<\lambda<1$. Note that this model reduces into the elliptical model if $\lambda=1$.

We extend the model to an asymmetrical formulation in a one-dimensional coordinate system $(x)$ by introducing a new parameter $x_{c}$ as the location of the maximum $\delta$ and repeating $a$ and $\lambda$ on either side of $x_{c}$,

$$
\delta(x)= \begin{cases}\delta_{-}^{\text {joint }}\left(\frac{x-x_{-}^{\text {tip }}}{x_{-}^{\text {joint }}-x_{-}^{\text {tip }}}\right)^{3 / 2} & , x_{-}^{\text {tip }}<x<x_{-}^{\text {joint }} \\ D\left[1-\left(\frac{x-x_{c}}{\lambda_{-} a_{-}}\right)^{2}\right]^{1 / 2} & , x_{-}^{\text {joint }} \leq x<0 \\ D\left[1-\left(\frac{x-x_{c}}{\lambda_{+} a_{+}}\right)^{2}\right]^{1 / 2} & , 0 \leq x \leq x_{+}^{\text {joint }} \\ \delta_{+}^{\text {joint }}\left(\frac{x_{+}^{\text {tip }}-x}{x_{+}^{\text {tip }}-x_{+}^{\text {joint }}}\right)^{3 / 2} & , x_{+}^{\text {joint }}<x<x_{+}^{\text {tip }} \\ 0 & , \text { otherwise }\end{cases}
$$

where $x_{c}$ is the location of maximum $\delta$ such that $\delta\left(x_{c}\right)=D, a_{ \pm}$are the rupture half-lengths on either side of $x_{c}, x_{ \pm}^{\text {tip }}=x_{c} \pm a_{ \pm}$are the locations of rupture tips, $\lambda_{ \pm}$controls the radius of the ellipse $a_{ \pm}^{\text {ellipse }}=\lambda_{ \pm} a_{ \pm}$, in which $0<\lambda_{ \pm}<1, x_{ \pm}^{\text {joint }}=x_{c} \pm\left(\sqrt{1+3 \lambda_{ \pm}^{2}}-1\right) a_{ \pm}$are the locations where $\delta(x)$ switches between elliptical and $\left( \pm\left[a_{ \pm}-x\right]\right)^{3 / 2}$ forms, and $\delta_{ \pm}^{\text {joint }}=$ $\delta\left(x_{ \pm}^{\text {joint }}\right)$, as shown in Fig. 4b. 


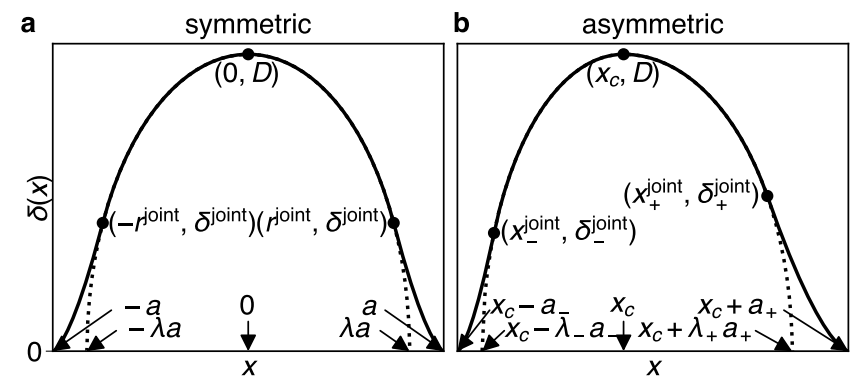

Figure 4. Parameters of the proposed slip profile model in one-dimensional (a) symmetric form (Eqn. 2) and (b) asymmetric form (Eqn. 3). (a) Dotted curve shows the elliptical model this model follows between $\pm r^{\text {joint }}$ with radius $\lambda a$ and height $\delta(0)=D$, in which $a$ is the half-length of the rupture and $0<\lambda<1$. (b) Dotted curve shows the asymmetric elliptical model this model follows between $x_{ \pm}^{\text {joint }}$ with radius $\lambda_{ \pm} a_{ \pm}$on either side of $x_{c}$, in which $x_{c}$ is the location such that $\delta\left(x_{c}\right)=D$, $x_{c} \pm a_{ \pm}$are the locations of rupture tips $x_{ \pm}^{\text {tip }}$.

\section{Results}

Fig. 5 shows slip profiles and associated stress changes measured from eight different contained laboratory-generated earthquakes and the respective model fits, where events (1)-(4) are completely contained and events (5)-(8) are partially contained. The spatial resolution of slip profile $\delta(x)$ measurements is arguably not high enough to resolve the fine details near the rupture tips. However, $\Delta \tau(x)$ is very sensitive to the details of $\delta(x)$ nonlocally, therefore measurements of $\Delta \tau(x)$ provide additional data to guide and resolve the fine details in $\delta(x)$ near the rupture tips. Simultaneously fitting a model to both $\delta(x)$ measurements and $\Delta \tau(x)$ measurements is a more robust way to resolve $\delta(x)$ and the associated $\Delta \tau(x)$ of earthquake ruptures compared to interpolating between sparse measurements.

Fig. 5b shows two relatively large rupture events (4) and (8) from our experiments to demonstrate the quality of model fits of the elliptical model, the bell-shaped model, and the proposed model. To accommodate the restriction that the bell-shaped model cannot be stretched asymmetrically, we sliced slip profiles in half with respect to the location of maximum $\delta$ for the comparison between models. All three models fit $\delta(x)$ well, however, the shapes of $\Delta \tau(x)$ differ near the rupture tip, i.e., in the earthquake arrest zone. Importantly, our $\Delta \tau(x)$ measurements nearly always contain at least one data point with an intermediate value of $\Delta \tau$ located between the maximum $\Delta \tau$ at the rupture tip and the nearly constant $\Delta \tau$ within the central portion of the ruptured region. Even though the spatial resolution 
a (1) FS01-038-7MPa-P-1-01

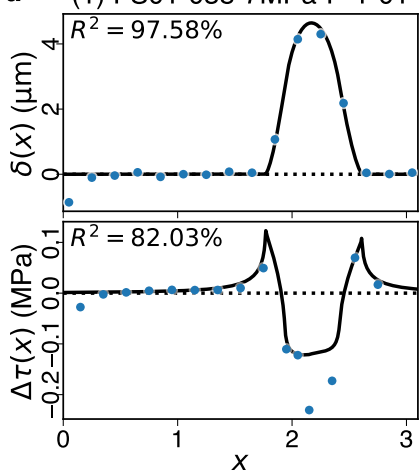

(5) FS01-039-7MPa-P-1-02

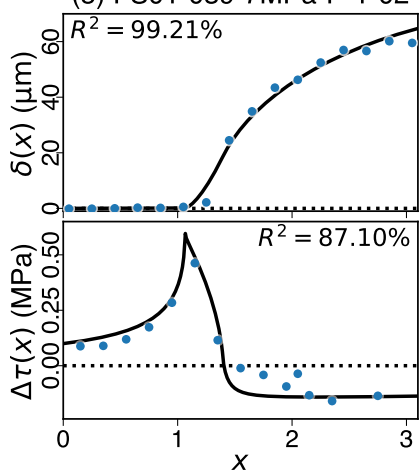

(2) FS01-040-4MPa-RP-1-02
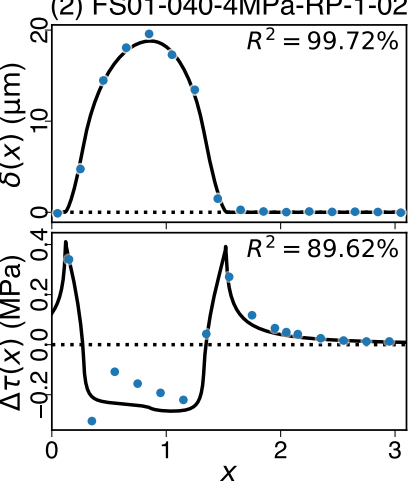

(6) FS01-040-4MPa-RP-1-05

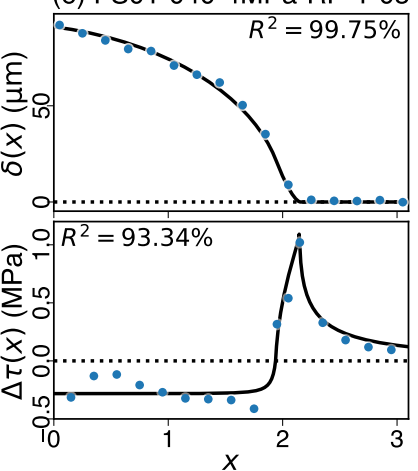

(3) FS01-040-4MPa-RP-2-03
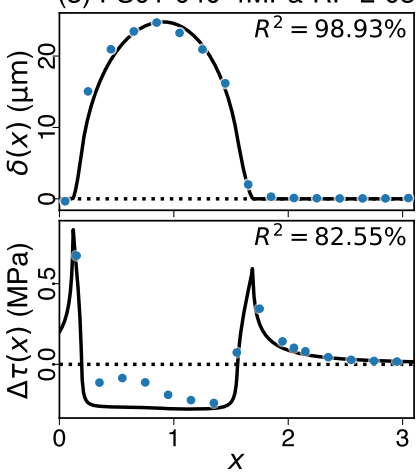

(7) FS01-040-4MPa-RP-1-06
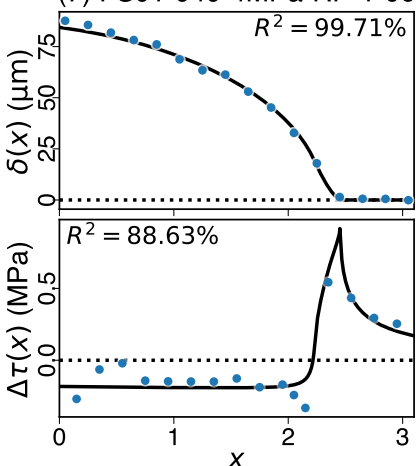

(4) FS01-040-4MPa-RP-4-05

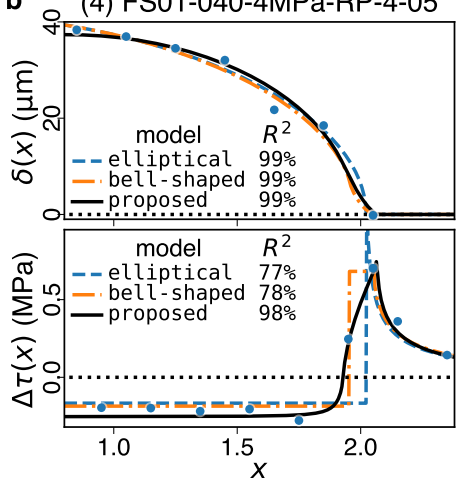

(8) FS01-040-4MPa-RP-4-06

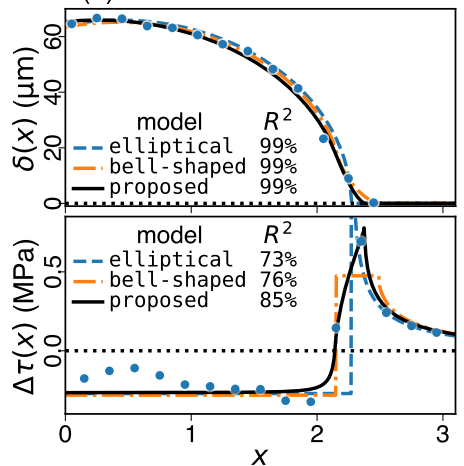

Figure 5. Examples of measured rupture events and model fits of (1)-(4) completely contained and (5)-(8) partially contained laboratory earthquakes. (a) Blue dots indicate measurements of $\delta(x)$ and $\Delta \tau(x)$. Solid curves are results of model fits. The coefficient of determination $R^{2}$ of model fits is marked next to each curve. (b) Comparison between the elliptical, the bell-shaped, and the proposed model. Entries in legends denote $R^{2}$ of each model fit, where only half of the rupture is shown.

of strain measurements is not high enough to verify the exact shape of $\Delta \tau(x)$ within the earthquake arrest zone, they provide clear evidence of the existence of an earthquake arrest zone and a smoothly varying $\Delta \tau(x)$ within the arrest zone. The proposed model better matches our data then the discontinuity in $\Delta \tau(x)$, which is a feature of both the elliptical model and the bell-shaped model. Of the 24 completely contained ruptures and 13 partially contained ruptures studied here, the coefficient of determination $R^{2}$ of $\delta(x)$ and $\Delta \tau(x)$ fits are $97.7 \% \pm 2.3 \%$ and $83.7 \% \pm 10.7 \%$, respectively. 


\section{Discussion}

\subsection{Earthquake arrest zone and comparison between different models}

The proposed model merges an elliptical slip profile with a $x^{3 / 2}$ form at the edges. This allows constant stress drop in the center while keeping the stress concentration at rupture tips finite, and retains a smooth transition in between. The linear tapering feature in slip profiles observed in natural faults is related to the existence of an earthquake arrest zone. Our model's earthquake arrest zone width is $w_{\mathrm{az}} \equiv 2\left(a-r^{\text {joint }}\right)=2 a\left(2-\sqrt{1+3 \lambda^{2}}\right)$, as shown in the shaded area in Fig. 1d. It is the region where $\delta(x)$ is approximately linear and where $\Delta \tau(x)$ transitions from the stress drop within the ruptured region, $\Delta \tau_{\min }$, to the maximum stress increase at the tips of the arrested rupture, $\Delta \tau_{\max }$. The earthquake arrest zone width $w_{\mathrm{az}}$ increases with rupture length $2 a$ but their ratio $w_{\mathrm{az}} / 2 a$ is a function of only the shape parameter $\lambda$, i.e., $w_{\mathrm{az}} / 2 a=2-\sqrt{1+3 \lambda^{2}}$. $w_{\mathrm{az}}$ vanishes if $\lambda=1$ and widens as $\lambda$ decreases.

The values of $\lambda$ that best fit laboratory measurements of completely contained ruptures ranged from 0.49 to 0.99 , with a median value of 0.85 which reflects $w_{\mathrm{az}} / 2 a \approx 20 \%$, consistent with field observations from larger earthquakes (Cowie \& Scholz, 1992; Scholz \& Lawler, 2004).

$w_{\mathrm{az}}$ in the proposed model is conceptually similar to the friction breakdown zone width of the bell-shaped model ( $s$ in Cowie and Scholz (1992), or $a-d$ in Bürgmann et al. (1994)). In the Walsh and Watterson (1987) model, the earthquake arrest zone is essentially the entire rupture half-length. This model also has the approximately linear tapering feature at the edges and limited stress concentration at rupture tips, but its associate stress changes have a fixed triangular shape that does not match our observations or other models that show roughly uniform $\Delta \tau(x)$ inside the ruptured region.

\subsection{Scaling of the earthquake arrest zone, earthquake stress drop, and seis- mically observed earthquake fracture energy $E_{\mathrm{G}}$}

Our experiments produce contained ruptures with half lengths that range from $0.5 \mathrm{~m}$ to $2 \mathrm{~m}$. By itself, this provides limited scaling information, and we observe no apparent trend in $D$ or $\lambda$ against rupture size. However, we gain important insights by imposing some physical constraints supported by field observations of large and small earthquakes. 
Earthquake ruptures range in size from hundreds of $\mathrm{km}$ to hundreds of $\mathrm{mm}$ while absolute strength and fracture energy of the rocks $\left(\tau_{\mathrm{p}}, \tau_{\mathrm{r}}, \Gamma\right)$ and stress levels of the crust $\left(\tau_{0}\right)$ should remain relatively scale-independent. Note that the apparent peak stress $\Delta \tau_{\max }+\tau_{0}(x)$ may not represent the actual peak strength of the interface $\tau_{\mathrm{p}}$. We expect $\Delta \tau_{\max }$ to be bounded by $\tau_{\mathrm{p}}-\tau_{0}(x)$. Therefore, $\Delta \tau_{\max }$ should also be scale-independent with respect to rupture size. These constraints agree with the most physically reasonable of the scaling scenarios considered by Cowie and Scholz (1992). We also assume that the average stress drop during an earthquake $\overline{\Delta \tau} \propto \mu D / a$ is scale independent, consistent with observations of large and small earthquakes (e.g., Hanks, 1977; Kanamori, Hiroo and Anderson, 1975; Baltay, Ide, Prieto, \& Beroza, 2011).

To illustrate the scaling mathematically, we analytically calculate $\Delta \tau_{\max }$ and $\Delta \tau_{\min }$ by plugging the slip profile into Eqn. 1 at the rupture tip and at the center. Assuming a symmetric crack, this results in

$$
\begin{aligned}
& \Delta \tau_{\max }=\Delta \tau(r=a)=\frac{\mu^{*}}{2 \pi} \frac{D}{a} \Lambda_{\mathrm{p}}(\lambda), \\
& \Delta \tau_{\min }=\Delta \tau(r=0)=-\frac{\mu^{*}}{2 \pi} \frac{D}{a} \Lambda_{\mathrm{r}}(\lambda),
\end{aligned}
$$

in which

$$
\begin{aligned}
\Lambda_{\mathrm{p}}(\lambda)= & -2 \frac{\theta}{\lambda}+\frac{3 \cos \theta}{\alpha^{3}}\left[2 \alpha-\sqrt{2} \tanh ^{-1}\left(\frac{\alpha}{\sqrt{2}}\right)\right] \\
& +\frac{2}{\lambda \beta}\left[\tan ^{-1}\left(\frac{\lambda+\gamma}{\beta}\right)-\tan ^{-1}\left(\frac{\lambda-\gamma}{\beta}\right)\right], \\
\Lambda_{\mathrm{r}}(\lambda)= & 2 \frac{\theta}{\lambda}+\frac{6 \cos \theta}{\alpha^{3}}\left[\tanh ^{-1}(\alpha)-\alpha\right],
\end{aligned}
$$

where $\alpha=\sqrt{1-\lambda \sin \theta}, \beta=\sqrt{1-\lambda^{2}}, \gamma=\tan (\theta / 2)$, and $\theta=\sin ^{-1}\left(\left(\sqrt{1+3 \lambda^{2}}-1\right) / \lambda\right)$. Namely, both $\Delta \tau_{\max }$ and $\Delta \tau_{\min }$ are proportional to $\mu^{*}$ and $D / a$. The stress ratio $\Delta \tau_{\max } /\left(-\Delta \tau_{\min }\right)$ $=\Lambda_{\mathrm{p}}(\lambda) / \Lambda_{\mathrm{r}}(\lambda)$ spans $(0,+\infty)$ for $\lambda \in(0,1)$, monotonically increases as $\lambda$ increases, and monotonically decreases as $w_{\mathrm{az}} / 2 a$ increases. This shows that the proposed model can adapt to arbitrary $\Delta \tau_{\max }$ and $\Delta \tau_{\min }$ as long as $\Delta \tau_{\max }>0>\Delta \tau_{\min }$, but might have limitations fitting both arbitrary stress ratio and arbitrary $w_{\mathrm{az}}$ since both depend on $\lambda$.

Imposing all the above constraints (scale independent $D / a, \Delta \tau_{\max }, \Delta \tau_{\min }$, and $\tau_{0}$ ) necessitates a scale invariant $\lambda$, which describes a self-similar slip profile, as shown in Fig. 6a. Note that Fig. 6 shows the scaling relations of independent arrested earthquake ruptures rather than snapshots of rupture growth. A result of self-similarity is that $w_{\mathrm{az}}$ scales with rupture length $(2 a)$, as shown in Fig. 6c, and this implies that the seismically observed 

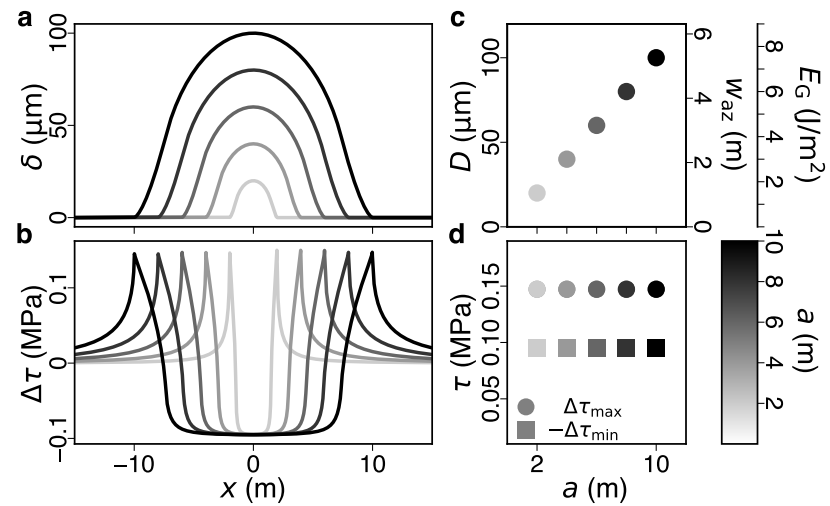

Figure 6. Scaling relations of the proposed model for $a$ from 2 to $10 \mathrm{~m}$. (a) Slip profile $\delta(x)$ of earthquake ruptures of different $a$ and (b) the respective associated stress changes $\Delta \tau(x)$. (c) Scaling relations of maximum slip $D$, earthquake arrest zone width $w_{\mathrm{az}}$, and seismically observed earthquake fracture energy $E_{\mathrm{G}}$ to $a$. (d) $\Delta \tau_{\max }$ and $-\Delta \tau_{\min }$ of different $a$.

earthquake fracture energy $E_{\mathrm{G}}$ increases with earthquake size, consistent with seismic observations (Abercrombie \& Rice, 2005). In our model with constant $\lambda, E_{\mathrm{G}} \propto \delta^{\text {joint }} \propto D$. Since $D / a$ is also a constant, $E_{\mathrm{G}} \propto a$. Note also that $E_{\mathrm{G}} \propto w_{\mathrm{az}}$ since both $w_{\mathrm{az}} \propto a$ and $E_{\mathrm{G}} \propto a$, for fixed $\lambda$.

The scaling relations described above are not unique to our proposed model; they are identical to those proposed for the bell-shaped model (Cowie \& Scholz, 1992) and similar to a recent theoretical model of dynamic ruptures (Weng \& Ampuero, 2019). The self-similar scaling is also consistent with the CFTT (constant fault tip taper) model (Scholz \& Lawler, 2004; Scholz, 2019), analogous to constant CTOA (crack tip opening angle) model for mode I fracture (Kanninen \& Popelar, 1985). Since our proposed model has a slip profile that is fairly close to a linear taper, we propose that it can be considered a first order analytical approximation to the CFTT model.

\subsection{Physical mechanisms underlying fault tip taper and earthquake arrest zone}

Field evidence shows that fault tip taper increases with stress at the rupture tip (Scholz \& Lawler, 2004). As a result, earthquakes that rupture a preexisting fault (with lower strength) taper more gradually than shear cracks that form new faults. This relationship 
can be derived analytically from our proposed model. We define that the fault tip taper

$$
F T T \equiv \frac{\delta^{\text {joint }}}{w_{\mathrm{az}} / 2}=\frac{D}{a} \frac{\cos \theta}{(1-\lambda \sin \theta)} \equiv \frac{D}{a} F(\lambda),
$$

where $\delta^{\text {joint }}=D \cos \theta, \theta=\sin ^{-1}\left(\left(\sqrt{1+3 \lambda^{2}}-1\right) / \lambda\right)$, and $F$ is a monotonically increasing function of $\lambda$. If $\lambda$ is constant, the maximum stress change, the minimum stress change, and their difference scales positively with $F T T$ since $F T T, \Delta \tau_{\max }$, and $\Delta \tau_{\min }$ are all proportional to $D / a$.

The linear taper of the slip profile has been thought to be due to inelastic deformation in the rock volume around the fault tips (Cowie \& Scholz, 1992; Bürgmann et al., 1994; Scholz \& Lawler, 2004) possibly over multiple earthquake ruptures (Walsh \& Watterson, 1987). However, our laboratory earthquake ruptures exhibit earthquake arrest zones but show no sign of off-fault damage, suggesting that the features observed in the earthquake arrest zone can result from either friction processes occurring at the interface, or some other mechanisms.

The approximately linear taper at the edges of $\delta(x)$ and, equivalently, the earthquake arrest zone observed from $\Delta \tau(x)$ measurements in our experiments is orders of magnitude larger than the length-scale of cohesive zones that result from commonly used friction laws, e.g., slip-weakening friction (Ida, 1972; Palmer \& Rice, 1973; Andrews, 1976) and rate- and state-dependent friction (Dieterich, 1979; Ruina, 1983), which also exhibit slipweakening behavior during dynamic rupture propagation (Cocco \& Bizzarri, 2002). While the averaged $w_{\mathrm{az}}$ of completely contained rupture events from our experiments was about $0.4 \mathrm{~m}$, fracture mechanics theory (Palmer \& Rice, 1973) predicts a cohesive zone width of $w_{\mathrm{coh}}=9 \pi K^{2} /\left[32\left(\tau_{\mathrm{p}}-\tau_{\mathrm{r}}\right)^{2}\right]=9 \pi E d_{0}^{2} /(128 \Gamma) \approx 10 \mathrm{~mm}$ with $\Gamma \approx 1 \mathrm{~J} / \mathrm{m}^{2}$ (Kammer \& McLaskey, 2019) and $d_{0}=1 \mu \mathrm{m}$, which is reasonable for the bare granite surfaces in our experiment. The $5 \mathrm{~mm}$ off-fault location of the strain gauges cannot explain this discrepancy. Furthermore, past experiments where both the top and bottom surfaces of the granite sample were instrumented with slip sensors showed that ruptures were generally one-directional, so it is unlikely that $2 \mathrm{D}$ effects associated with the $0.3 \mathrm{~m}$ thickness of the granite sample strongly affect our estimates.

We argue that the earthquake arrest zone observed in our experiments and the corresponding linear taper that has been mapped in field studies are primarily the result of a heterogeneous initial stress $\tau_{0}(x)$ prior to rupture and does not relate directly to the strength evolution of the interface. Under uniform stress, strength, and fracture energy $\Gamma$, fracture 
mechanics predicts that crack growth will not slow down once it initiates. Therefore, in order to stop an earthquake rupture, the rupture front must encounter either a barrier with high fracture energy $\Gamma(x)$ or unfavorable stress conditions, i.e., $\tau_{0}(x)<\tau_{\mathrm{r}}$. Even though previous studies (e.g., Abercrombie \& Rice, 2005; Viesca \& Garagash, 2015; Cocco et al., 2016; Nielsen et al., 2016) have reported scale-dependent "earthquake fracture energy" $E_{\mathrm{G}}$, $\Gamma(x)$ is considered a scale-independent material or interfacial property (e.g., Day et al., 2005). We believe that the most likely reason for rupture termination is propagation into unfavorable stress conditions, at least for earthquakes rupturing preexisting faults (Ke et al., 2018). This is similar to the idea of rupture interacting with the stress shadow of a previous earthquake on the same fault (e.g., Gupta \& Scholz, 2000). As illustrated by the dynamic rupture simulations described in section 6.4 , we suggest that the $\Delta \tau(x)$ in the earthquake arrest zone, the $\Delta \tau-\delta$ relations of Fig. 1e, and the corresponding linear taper in $\delta(x)$ are the result of rupture termination conditions and bear little resemblance to the underlying friction behavior of the material or interface. The stress changes within the earthquake arrest zone $\Delta \tau(x)$ mainly reflect the transition of $\tau_{0}(x)$ from above to below $\tau_{\mathrm{r}}$. Large earthquakes appear to have large $w_{\mathrm{az}}$ and large seismically observed fracture energy $E_{\mathrm{G}}$ because they must propagate further into unfavorable stress conditions to halt rupture. It is possible that the scale dependency of $E_{\mathrm{G}}$ could result from the scale-dependent earthquake arrest zone while $\Gamma$ remains scale-independent.

\subsection{Examples of heterogeneous $\tau_{0}(x)$ as the source of observed earthquake arrest zone features}

To test the above conjecture that the seismically inferred $\Delta \tau-\delta$ relationship can be the result of heterogeneous $\tau_{0}(x)$, we simulated fully dynamic rupture propagation and termination with the spectral boundary integral method (Breitenfeld \& Geubelle, 1998) in two different initial stress distributions $\tau_{0}(x)$. The first example (Fig 7 ) has a trapezoidal $\tau_{0}(x)$, shown as the black dashed line in Fig $7 \mathrm{c}$, and the resulting slip distribution and stress changes emulate the features of the earthquake arrest zone that we observed in the laboratory experiments. The second example (Fig 8) has a boxcar $\tau_{0}(x)$, shown as the black dashed line in Fig 8c, to emulate the earthquake arrest zone with a constant stress change. Both simulations have identical material properties $\left(E=30 \mathrm{GPa}, \nu=0.23, \rho=2700 \mathrm{~kg} / \mathrm{m}^{3}\right)$ and linear slip-weakening strength evolution law $\tau_{\mathrm{s}}(\delta)$ with peak strength $\tau_{\mathrm{p}}=8 \mathrm{MPa}$, residual strength $\tau_{\mathrm{r}}=6 \mathrm{MPa}$, and critical slip distance $d_{0}=1 \mu \mathrm{m}$. The dynamic rupture is nucleated 


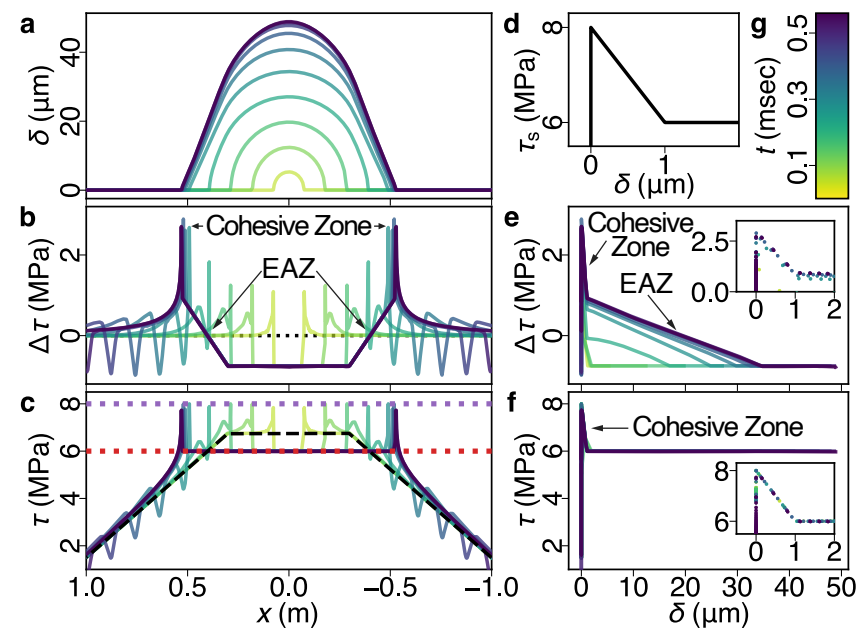

Figure 7. Dynamic simulation of an initial shear stress distribution $\tau_{0}(x)$ that results in linear slip taper. (a) Snapshots of the slip profile $\delta(x)$, (b) the respective associated stress changes $\Delta \tau(x)$, and (c) the respective absolute stress $\tau(x)$ of the dynamic rupture at different times color coded with $(\mathrm{g})$, in which the opaque dark blue curves represent the static outcomes. Black dashed line in (c) shows a trapezoidal initial stress distribution $\tau_{0}(x)$. Purple and red dotted lines show the peak strength and the residual strength levels. Oscillations in both (b) and (c) are the shear wave emitted from the nucleation. (d) depicts the imposed strength evolution law $\tau_{\mathrm{s}}(\delta)$. (e) and (f) show the resultant $\Delta \tau-\delta$ and $\tau-\delta$ relationships at different times, respectively. Insets are zoomed in at the spike near $\delta=0$. The inset in (f) strictly follows $\tau_{\mathrm{s}}(\delta)$ shown in (d). The extent of linear slip taper in (a) coincides with the linear transition from $\Delta \tau_{\min }$ to $\Delta \tau_{\max }$ in (b).

by manually extending a seed crack (dropping $\tau_{\mathrm{p}}$ to $\tau_{\mathrm{r}}$ ) bilaterally from $x=0$ at half the Rayleigh wave speed until it reaches the critical crack length $L_{\mathrm{c}} \approx 36 \mathrm{~mm}$ and becomes unstable spontaneously. The rupture front then accelerates towards the Rayleigh wave speed and decelerates once it propagates into unfavorable stress states, i.e., $\tau_{0}(x)<\tau_{\mathrm{r}}(x)$. When the rupture runs out of available strain energy to release, it spontaneously arrests.

The difference between Fig. 7e and Fig. 7f, and similarly between Fig. 8e and Fig. 8f, demonstrates the distinction between the $\Delta \tau-\delta$ that can be inferred from earthquake observations and the underlying frictional strength evolution $\tau_{\mathrm{s}}-\delta$ relationship. Since the absolute stress level $\tau$ in the Earth is mainly inaccessible, the measured or inferred $\Delta \tau$ from earthquakes was sometimes thought to represent the absolute stress level by assuming $\tau_{0}(x)$ is uniform across the extent of a rupture. With these two examples, we demonstrated that the apparent features in $\delta(x)$, such as linear taper or bell-shaped, and in $\Delta \tau$, such as smooth 

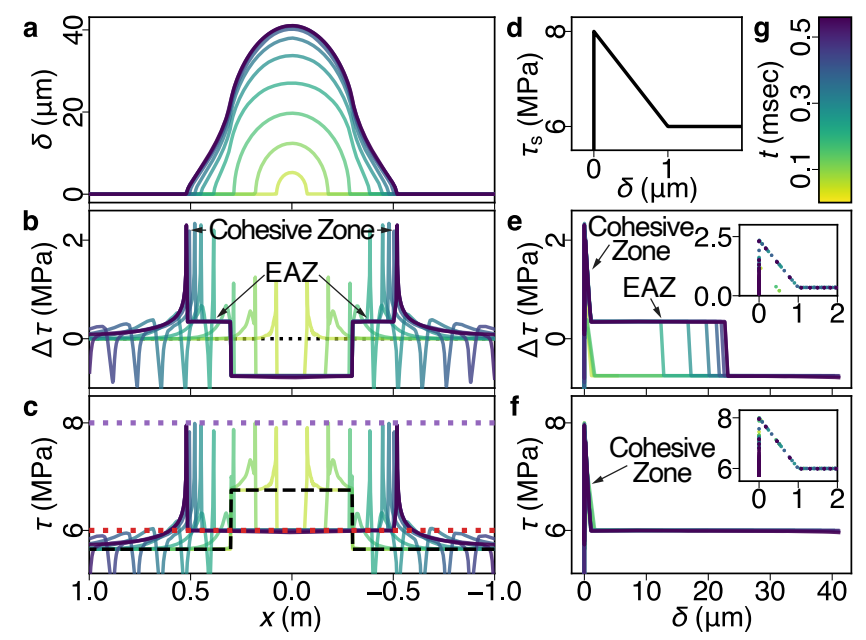

Figure 8. Dynamic simulation of an initial shear stress distribution $\tau_{0}(x)$ that results in a bellshaped slip profile. All panels are similar to Fig. 7. Black dashed line in (c) shows a boxcar initial stress distribution $\tau_{0}(x)$. Note that $\tau_{0}(x)<\tau_{\mathrm{r}}$ outside the boxcar.

transition or a sudden step, can solely result from the shape of the initial stress distribution $\tau_{0}(x)$. While the actual cohesive zone is small and hard to measure, the apparent large-scale feature of $\Delta \tau(x)$ in the arrest zone (Fig $7 \mathrm{~b}$ ) and the apparent slip-weakening feature in the $\Delta \tau-\delta$ curve (Fig $7 \mathrm{e}$ ) can be produced by the heterogeneous $\tau_{0}(x)$ and the misrepresentation of $\Delta \tau$ as $\tau_{\mathrm{s}}$. Similarly, with a crafted $\tau_{0}(x)$ (Fig 8c), we can emulate a bell-shaped slip profile (Fig 8a) with linear elasticity (and no off-fault damage).

\subsection{Smooth observed slip profile}

Our contained laboratory-generated earthquakes have smoother slip profiles compared to natural earthquakes. This could be because the simulated fault is more smooth and flat than natural faults. Another possibility is that our experiments produced "baby" earthquakes that reached unfavorable stress conditions and terminated soon after nucleating and before the rupture front was fully dynamic (Svetlizky et al., 2017), and therefore more complex high-speed processes could not engage. Measurements of $\Delta \tau(x)$ inside the ruptured region, where dynamic rupture propagation took place, were slightly more deviated from the model and less smooth compared to the rest of the fault, as shown in Fig. 5. Perhaps the ruptures are not completely homogeneous along depth while the strain gages are glued on the surface of the rock blocks, or some randomness is introduced by the rapid fluctuations 
in slip and stress during the dynamic rupture process as seen in a previous study with a similar experimental setup (McLaskey et al., 2015).

\section{Conclusions}

Our contained laboratory-generated earthquakes ruptured a nominally flat and smooth frictional interface free from heterogeneities in geometry and material properties. We found that heterogeneity in initial stress distribution was sufficient to generate laboratory earthquakes that terminated within the 3-meter long simulated fault, providing a rare opportunity to study the features of slip profiles and the associated stress changes in a simplified laboratory setting. In addition to local slip measurements, we used local shear strain measurements to help resolve the details of slip profiles near the rupture tips, where stress changes are profound. Near the rupture tips, we consistently observe an earthquake arrest zone where stress changes smoothly transition from the maximum level at the crack tip to the minimum level within the ruptured region. The earthquake arrest zone was $0.06 \mathrm{~m}$ to $0.95 \mathrm{~m}$ in size and was, on average, about $20 \%$ of the overall rupture length, consistent with field observations of constant slip gradients (Cowie \& Scholz, 1992; Scholz \& Lawler, 2004). However the size of the arrest zone we observe is orders of magnitude larger than the cohesive zone predicted by fracture mechanics theory, using reasonable values of friction parameters. This leads us to believe that the observed features in the arrest zone are primarily produced by the heterogeneous initial stress distribution required to stop an earthquake rupture rather than the fault strength. Using a set of numerical simulations of spontaneous dynamic rupture propagation and termination, we illustrated how an earthquake's stress change versus slip relationship $(\Delta \tau-\delta)$ inferred from static stress changes can be profoundly different from the underlying frictional strength evolution $\left(\tau_{\mathbf{s}}-\delta\right)$. This has profound implications for how seismically derived estimates of certain earthquake parameters should be interpreted: The seismically inferred increase in fracture energy $E_{\mathrm{G}}$ and critical slip distance $d_{0}$ with increasing earthquake size (e.g., Abercrombie \& Rice, 2005) reflects the manner in which earthquake ruptures arrest rather than the way fault strength evolves with slip.

We propose a slip profile model that does not contain the stress singularity of the elliptical model; it has an earthquake arrest zone that moderates stress changes at the rupture tip. Different from previous models that also include an earthquake arrest zone, such as the bell-shaped model (Cowie \& Scholz, 1992; Bürgmann et al., 1994), our proposed model features smoothly varying stress changes that are more compatible with our laboratory 
measurements, and this facilitates the interpretation of the extent of the earthquake arrest zone that is otherwise difficult to define. While the full details of the stress changes within the earthquake arrest zone are not resolved due to limited spatial resolution in our experimental measurements, the inferred model provides a proper first-order approximation to the smooth transition from the maximum to the minimum level through a mathematically simple and numerically stable formulation of the slip profile. Constrained by physical measurements, the model may be useful as a component of more complicated fault rupture and rupture sequence earthquake models.

\section{Acknowledgments}

This research was supported by the National Science Foundation under grant EAR-1763499. Data used in this paper were acquired during laboratory experiments conducted at Cornell University. Data reported here are publicly available at https://eCommons.cornell.edu. We thank C. Scholz for a helpful review that stimulated transformative changes to this paper. We thank H. Weng, and two anonymous reviewers for their helpful reviews. We thank Bill $\mathrm{S}$. Wu for assistance with the experiments. The authors declare that they have no conflict of interest.

\section{References}

Abercrombie, R. E., \& Rice, J. R. (2005). Can observations of earthquake scaling constrain slip weakening? Geophysical Journal International, 162(2), 406-424. doi: 10.1111/ j.1365-246X.2005.02579.x

Andrews, D. J. (1976). Rupture propagation with finite stress in antiplane strain. Journal of Geophysical Research, $81(20), 3575$. doi: 10.1029/JB081i020p03575

Baltay, A., Ide, S., Prieto, G., \& Beroza, G. (2011). Variability in earthquake stress drop and apparent stress. Geophysical Research Letters, 38(6), 1-6. doi: 10.1029/ 2011GL046698

Bilby, B. A., \& Eshelby, J. D. (1968). Dislocations and the theory of fracture. In H. Liebowitz (Ed.), Fracture, an advanced treatise (Vol. I, pp. 99-182). San Diego, Calif.: Academic.

Breitenfeld, M. S., \& Geubelle, P. H. (1998). Numerical analysis of dynamic debonding under 2D in-plane and 3D loading. International Journal of Fracture, 93(1/4), 13-38. doi: $10.1023 /$ A:1007535703095 
Bürgmann, R., Pollard, D. D., \& Martel, S. J. (1994). Slip distributions on faults: effects of stress gradients, inelastic deformation, heterogeneous host-rock stiffness, and fault interaction. Journal of Structural Geology, 16(12), 1675-1690. doi: 10.1016/0191 $-8141(94) 90134-1$

Cocco, M., \& Bizzarri, A. (2002). On the slip-weakening behavior of rate- and state dependent constitutive laws. Geophysical Research Letters, 29(11), 11-1-11-4. doi: 10.1029/2001GL013999

Cocco, M., Tinti, E., \& Cirella, A. (2016). On the scale dependence of earthquake stress drop. Journal of Seismology, 20(4), 1151-1170. Retrieved from http://link.springer .com/10.1007/s10950-016-9594-4 doi: 10.1007/s10950-016-9594-4

Cowie, P. A., \& Scholz, C. H. (1992). Physical explanation for the displacement-length relationship of faults using a post-yield fracture mechanics model. Journal of Structural Geology, 14(10), 1133-1148. doi: 10.1016/0191-8141(92)90065-5

Dawers, N. H., Anders, M. H., \& Scholz, C. H. (1993). Growth of normal faults: Displacement-length scaling. Geology, 21(12), 1107. doi: 10.1130/0091-7613(1993) 021<1107:GONFDL $\rangle 2.3 . \mathrm{CO} ; 2$

Day, S. M., Dalguer, L. A., Lapusta, N., \& Liu, Y. (2005). Comparison of finite difference and boundary integral solutions to three-dimensional spontaneous rupture. Journal of Geophysical Research: Solid Earth, 110(12), 1-23. doi: 10.1029/2005JB003813

Dieterich, J. H. (1979). Modeling of rock friction: 1. Experimental results and constitutive equations. Journal of Geophysical Research, 84(B5), 2161. doi: 10.1029/ JB084iB05p02161

Dudgale, D. (1960). Yielding of steel sheets containing slits. Journal of the Mechanics and Physics of Solids, 8(2), 100-104. doi: 10.1016/0022-5096(60)90013-2

Freed, A. M. (2005). Earthquake Triggering By Static, Dynamic, and Postseismic Stress Transfer. Annual Review of Earth and Planetary Sciences, 33(1), 335-367. doi: 10.1146/annurev.earth.33.092203.122505

Freund, L. B. (1990). Dynamic Fracture Mechanics (Vol. 25) (No. 9). Cambridge: Cambridge University Press. doi: 10.1017/CBO9780511546761

Gupta, A., \& Scholz, C. H. (2000). A model of normal fault interaction based on observations and theory. Journal of Structural Geology, 22(7), 865-879. doi: 10.1016/ S0191-8141(00)00011-0

Hanks, T. C. (1977). Earthquake stress drops, ambient tectonic stresses and stresses that 
drive plate motions. pure and applied geophysics: pageoph, 115(1-2), 441.

Ida, Y. (1972). Cohesive force across the tip of a longitudinal-shear crack and Griffith's specific surface energy. Journal of Geophysical Research, $77(20), 3796-3805$. doi: 10.1029/JB077i020p03796

Kammer, D. S., \& McLaskey, G. C. (2019). Fracture energy estimates from large-scale laboratory earthquakes. Earth and Planetary Science Letters, 511, 36-43. doi: 10 $.1016 /$ j.epsl.2019.01.031

Kanamori, H., \& Rivera, L. (2006). Energy partitioning during an earthquake. Geophysical Monograph Series, 170, 3-13. doi: 10.1029/170GM03

Kanamori, Hiroo and Anderson, D. L. (1975). Theoretical basis of some empirical relations in seismology. Bulletin of the Seismological Society of America, 65(5), 1073-1095.

Kanninen, M. F., \& Popelar, C. H. (1985). Advanced Fracture Mechanics. Oxford University Press.

Ke, C.-Y., McLaskey, G. C., \& Kammer, D. S. (2018). Rupture Termination in LaboratoryGenerated Earthquakes. Geophysical Research Letters, 45(23), 12784-12792. doi: 10.1029/2018GL080492

Manighetti, I., Campillo, M., Sammis, C., Mai, P. M., \& King, G. (2005). Evidence for selfsimilar, triangular slip distributions on earthquakes: Implications for earthquake and fault mechanics. Journal of Geophysical Research, 110(B5), B05302. doi: 10.1029/ 2004JB003174

Manighetti, I., King, G. C. P., Gaudemer, Y., Scholz, C. H., \& Doubre, C. (2001). Slip accumulation and lateral propagation of active normal faults in Afar. Journal of Geophysical Research: Solid Earth, 106(B7), 13667-13696. doi: 10.1029/2000JB900471

McLaskey, G. C. (2019). Earthquake Initiation From Laboratory Observations and Implications for Foreshocks. Journal of Geophysical Research: Solid Earth, 124(12), 12882-12904. doi: 10.1029/2019JB018363

McLaskey, G. C., Kilgore, B. D., \& Beeler, N. M. (2015). Slip-pulse rupture behavior on a 2 m granite fault. Geophysical Research Letters, 42(17), 7039-7045. doi: 10.1002/ 2015GL065207

Muraoka, H., \& Kamata, H. (1983). Displacement distribution along minor fault traces. Journal of Structural Geology, 5(5), 483-495. doi: 10.1016/0191-8141(83)90054-8

Nicol, A., Watterson, J., Walsh, J. J., \& Childs, C. (1996). The shapes, major axis orientations and displacement patterns of fault surfaces. Journal of Structural Geology, 
18(2-3), 235-248. doi: 10.1016/S0191-8141(96)80047-2

Nielsen, S., Spagnuolo, E., Smith, S. A., Violay, M., Di Toro, G., \& Bistacchi, A. (2016). Scaling in natural and laboratory earthquakes. Geophysical Research Letters, 43(4), 1504-1510. doi: 10.1002/2015GL067490

Palmer, A. C., \& Rice, J. R. (1973). The Growth of Slip Surfaces in the Progressive Failure of Over-Consolidated Clay. Proceedings of the Royal Society A: Mathematical, Physical and Engineering Sciences, 332(1591), 527-548. doi: 10.1098/rspa.1973.0040

Perry, S. M., Lambert, V., \& Lapusta, N. (2020). Nearly Magnitude-Invariant Stress Drops in Simulated Crack-Like Earthquake Sequences on Rate-and-State Faults with Thermal Pressurization of Pore Fluids. Journal of Geophysical Research: Solid Earth, 125(3). doi: 10.1029/2019JB018597

Rice, J. R. (1968, jun). A Path Independent Integral and the Approximate Analysis of Strain Concentration by Notches and Cracks. Journal of Applied Mechanics, 35(2), 379-386. Retrieved from https://asmedigitalcollection.asme.org/ appliedmechanics/article/35/2/379/392117/A-Path-Independent-Integral -and-the-Approximate doi: 10.1115/1.3601206

Ruina, A. (1983). Slip instability and state variable friction laws. Journal of Geophysical Research: Solid Earth, 88(B12), 10359-10370. doi: 10.1029/JB088iB12p10359

Scholz, C. H. (2019). The Mechanics of Earthquakes and Faulting (3rd ed.). Cambridge University Press. doi: 10.1017/9781316681473

Scholz, C. H., \& Lawler, T. M. (2004). Slip tapers at the tips of faults and earthquake ruptures. Geophysical Research Letters, 31(21), 1-4. doi: 10.1029/2004GL021030

Svetlizky, I., Albertini, G., Cohen, G., Kammer, D. S., \& Fineberg, J. (2020). Dynamic fields at the tip of sub-Rayleigh and supershear frictional rupture fronts. Journal of the Mechanics and Physics of Solids, 137, 103826. doi: 10.1016/j.jmps.2019.103826

Svetlizky, I., \& Fineberg, J. (2014). Classical shear cracks drive the onset of dry frictional motion. Nature, 509(7499), 205-208. doi: 10.1038/nature13202

Svetlizky, I., Kammer, D. S., Bayart, E., Cohen, G., \& Fineberg, J. (2017). Brittle Fracture Theory Predicts the Equation of Motion of Frictional Rupture Fronts. Physical Review Letters, 118(12), 125501. doi: 10.1103/PhysRevLett.118.125501

Uenishi, K., \& Rice, J. R. (2003). Universal nucleation length for slip-weakening rupture instability under nonuniform fault loading. Journal of Geophysical Research: Solid Earth, 108(B1), 2042. doi: 10.1029/2001JB001681 
Viesca, R. C., \& Garagash, D. I. (2015). Ubiquitous weakening of faults due to thermal pressurization. Nature Geoscience, 8(11), 875-879. doi: 10.1038/ngeo2554

Walsh, J. J., \& Watterson, J. (1987). Distributions of cumulative displacement and seismic slip on a single normal fault surface. Journal of Structural Geology, 9(8), 1039-1046. doi: $10.1016 / 0191-8141(87) 90012-5$

Weng, H., \& Ampuero, J. P. (2019). The Dynamics of Elongated Earthquake Ruptures. Journal of Geophysical Research: Solid Earth, 124(8), 8584-8610. doi: 10.1029/ 2019JB017684

Wu, B. S., \& McLaskey, G. C. (2019). Contained Laboratory Earthquakes Ranging from Slow to Fast. Journal of Geophysical Research: Solid Earth, 108(B1), 2019JB017865. doi: 10.1029/2019JB017865 